\title{
Spin Ladder with Anisotropic Ferromagnetic Legs in a Transverse Magnetic Field
}

\author{
G. I. Japaridze ${ }^{1}$, A. Langari ${ }^{2,3}$, and S. Mahdavifar ${ }^{4}$ \\ ${ }^{1}$ International Center for Condensed matter Physics, Universidade de Brasilia, \\ 70904-910 Brasilia-DF, Brazil and Andronikashvili Institute of Physics, \\ Tamarashvili str. 6, 0177, Tbilisi, Georgia \\ 2 Physics Department, Sharif University of Technology, Tehran 11365-9161, Iran \\ ${ }^{3}$ Institute for Studies in Theoretical Physics and Mathematics (IPM), Tehran \\ 19395-5531, Iran \\ ${ }^{4}$ Institute for Advanced Studies in Basic Sciences, Zanjan 45195-1159, Iran
}

\begin{abstract}
.
We study the ground state phase diagram of a two-leg spin ladder with anisotropic ferromagnetic leg couplings under the influence of a symmetry breaking transverse magnetic field by the exact diagonalization technique. In the case of antiferromagnetic coupling between legs we identified two phase transitions in the plane of magnetic field vs interchain coupling strength. The first one corresponds to the transition from the gapped rung-singlet phase to the gapped stripe-ferromagnetic phase. The second one represents the transition from the gapped stripe-ferromagnetic phase into the fully polarized ferromagnetic phase.
\end{abstract}

PACS numbers: 75.10.Jm Quantized spin models, 75.10.Pq Spin chain models 


\section{Introduction}

There has been recently considerable interest in study of the magnetic field-induced effects in low-dimensional quantum spin systems. In particular, critical properties of the spin $S=1 / 2$ isotropic antiferromagnetic two-leg ladders in a magnetic field have been a field of intense studies. This seems pertinent in the face of great progress made within the last years in fabrication of such ladder compounds [1]. Moreover, since the antiferromagnetic two-legs ladder systems have a gap in the spin excitation spectrum, they reveal extremely rich quantum behavior in the presence of magnetic field (See for recent review [2]). Such quantum phase transitions in spin systems with gapped excitation spectrum were indeed studied experimentally [3-8]. On the theoretical side these transitions were intensively investigated using different analytical and numerical techniques [9-23].

Ladder systems with ferromagnetic legs are much less studied. Partly, the reason is that spin-ladders with ferromagnetic legs are not still fabricated. However, from the theoretical point of view these systems are extremely interesting, since they open a new wide polygon for the study of complicated quantum behavior, unsuspected in more conventional spin systems $[9,24-30]$. The variety of open possibilities is clearly seen from the weak-coupling phase diagram of a two-leg ferromagnetic ladder which is a function of the intraleg exchange anisotropy $(\Delta)$ and the isotropic interleg coupling $\left(J_{\perp}\right)$ [28], it is presented in Fig 1. In addition to the fully gapped rung-singlet phase (RS) (common in the case of antiferromagnetic ladder [9, 10]), the ground state phase diagram contains the gapless Spin-Luttinger-Liquid phase with the easy-plane anisotropy (SLL) and the stripe-ferromagnetic (SFM) phase which are realized only in the case of ferromagnetic $\operatorname{legs}(\Delta>0)[9,28]$.

The very rich and interesting new phenomena arise in the case of competing ferromagnetic intraleg and antiferromagnetic interleg couplings, in particular in the presence of symmetry breaking magnetic field [28, 29]. The effect of the uniform magnetic field, applied parallel to $Z$ (quantization) axes, on the properties of the two-leg ladder systems was first studied (in the case of equivalent spin $S=1$ Heisenberg chain model) by H. Schulz [9]. In the case of gapped rung-singlet phase the magnetization appears only at finite critical value of the magnetic field equal to the spin gap [9, 12. This behavior is generic for the spin gapped $U(1)$ symmetric systems in a magnetic field which leaves the in-plane rotational invariance unchanged [30] and belongs to the universality class of the commensurate-incommensurate (C-IC) transitions [31].

The effect of an uniform transverse field in the case of $U(1)$ symmetric phase is highly nontrivial. In the case of classical spin chains this effect has been studied already a decade ago [32. However, in the case of quantum antiferromagnetic $X X Z$ chain this problem is still the subject of intense recent studies [32-41].

In this paper, we address the similar problem and study the effect of uniform transverse magnetic field on the ground state phase diagram of a two-leg ladder with anisotropic, ferromagnetically interacting legs coupled by antiferromagnetic interleg 


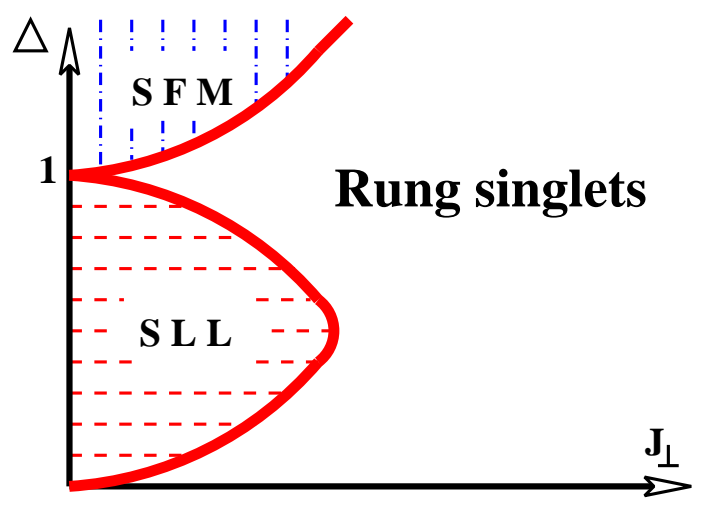

Figure 1. Schematic picture of the ground state phase diagram of the two-leg ladder as a function of the intraleg exchange anisotropy $(\Delta)$ and isotropic interleg coupling $\left(J_{\perp}\right)$ in the considered in this paper sector of the phase diagram corresponding to the competing ferromagnetic intraleg $(\Delta>0)$ and interleg antiferromagnetic $\left(J_{\perp}>0\right)$ exchange. The Spin-Luttinger-Liquid phase is denoted by SLL, while the StripeFerromagnetic phase - by SFM. (From Ref [28].)

exchange. The Hamiltonian of the model under consideration is given by

$$
H=H_{\text {leg }}^{(1)}+H_{\text {leg }}^{(2)}+H_{\perp}
$$

where the Hamiltonian for leg " $j "$ is

$$
H_{l e g}^{(j)}=-J \sum_{n=1}^{N}\left[\left(s_{j, n}^{x} s_{j, n+1}^{x}+s_{j, n}^{y} s_{j, n+1}^{y}+\Delta s_{j, n}^{z} s_{j, n+1}^{z}\right)+\mathrm{h}^{\mathrm{ext}} s_{j, n}^{x}\right]
$$

and the interleg coupling is

$$
H_{\perp}=J_{\perp} \sum_{j=1}^{N}\left(s_{1, j}^{x} s_{2, j}^{x}+s_{1, j}^{y} s_{2, j}^{y}+s_{1, j}^{z} s_{2, j}^{z}\right) .
$$

Here $s_{j, n}^{x, y}$ are spin $s=1 / 2$ operators on the $n$-th rung, and the index $j=1,2$ denotes the ladder legs. The intraleg coupling constant is ferromagnetic, $J>0$, and therefore the limiting case of isotropic ferromagnetic legs corresponds to $\Delta=1$ and the external magnetic field is proportional to $h=J \mathrm{~h}^{e x t}$.

The model (1) has been studied recently using the continuum-limit bosonization approach [28, 29]. The main attention in this studies was focused on the investigation of new field induced effects in the sector of phase diagram corresponding to the gapless easy-plane phase, present in the case of weak antiferromagnetic intraleg exchange $0<J_{\perp}<J$ [9, 28]. It has been shown, that in the presence of an infinitesimally small transverse magnetic field which breaks the in-plane rotational symmetry the gapless phase is unstable towards the gapped stripe-ferromagnetic (SFM) phase [29]. The SFM phase is characterized by the uniform magnetization in the direction of applied field and opposite magnetization of legs in the in-plane direction perpendicular to the field. It has been also shown, that when the magnetic field exceeds some critical value, the interleg antiferromagnetic order disappear and the system passes into the fully polarized ferromagnetic phase [29]. 
In the opposite case of strong rung exchange $J_{\perp} \gg J$ an analytical description of the magnetic properties of the system in the presence of transverse magnetic field is available only in the $S U(2)$ symmetric case at $\Delta=1$. In this limiting case it has been shown that the isotropic ladder in a magnetic field shows two second order phase transitions: at $\mathrm{h}^{e x t}=\mathrm{h}_{c 1}^{e x t}$ from a spin gapped rung-singlet (RS) phase to a gapless Spin-Luttinger-Liquid (SLL) phase and at $\mathrm{h}^{e x t}=\mathrm{h}_{c 2}^{e x t}$ a transition from a SLL phase into the fully polarized ferromagnetic (FM) phase (see Fig_2 a) 29].

In the case of finite intraleg exchange anisotropy $(\Delta \neq 1)$, when the effect of symmetry breaking transverse field is important, an analytical solution is not available. However, based on the qualitative estimations and symmetry analysis it has been shown that the two-step transition from the RS into the FM phase is present also in the case of ladder with anisotropic legs [29. In the same work it has been also predicted that for $\Delta \neq 1$ the RS phase is separated from the FM phase by the gapped SFM phase (see Fig $2 \mathrm{~b})$.

In this paper we continue our studies of the anisotropic spin ladder in the transverse magnetic field. In particular we apply the modified Lanczos method to diagonalize numerically finite $(N=12,16,20,24)$ ladder systems. Using the exact diagonalization results, we calculate the spin gap, magnetization, various spin-structure factors and the rung dimerization order parameter as a function of applied transverse field for various values of the anisotropy parameter $\Delta \neq 1$. Based on the exact diagonalization results we obtain the magnetic phase diagram of the ferromagnetic ladders with anisotropic legs showing two phase transitions in the plane of magnetic field vs interchain coupling in agreement with the predictions made in Ref. [27].

The outline of the paper is as follows: In section II we briefly discuss the model in the strong rung coupling limit and derive the effective spin chain Hamiltonian to outline the symmetry aspects of the problem. In section III we present the results of exact diagonalization calculations using the modified Lanczos method. Finally we conclude and summarize our results in section IV.

\section{Large rung coupling results}

In this section we briefly discuss the model (1) in the limiting case of strong rung coupling $J_{\perp} \gg J$. In this limit the model (1) can be mapped onto the effective spinchain Hamiltonian [4, 16]. This allows to outline the symmetry aspects of the problem under consideration.

At $J_{\perp} \gg J$ it is convenient to discuss the model by representing the site-spin algebra in terms of on-bond-spin operators [42. Indeed an individual rung may be in a singlet or a triplet state with corresponding spectrum given by

$$
E_{ \pm}=\left(\frac{J_{\perp}}{4} \pm h^{e x t}\right), E_{0}=\frac{J_{\perp}}{4}, E_{s}=-\frac{3 J_{\perp}}{4} .
$$

At $h^{e x t} \leq J_{\perp}$, one component of the triplet becomes closer to the singlet ground state such that for a strong enough magnetic field we have a situation that the singlet and 


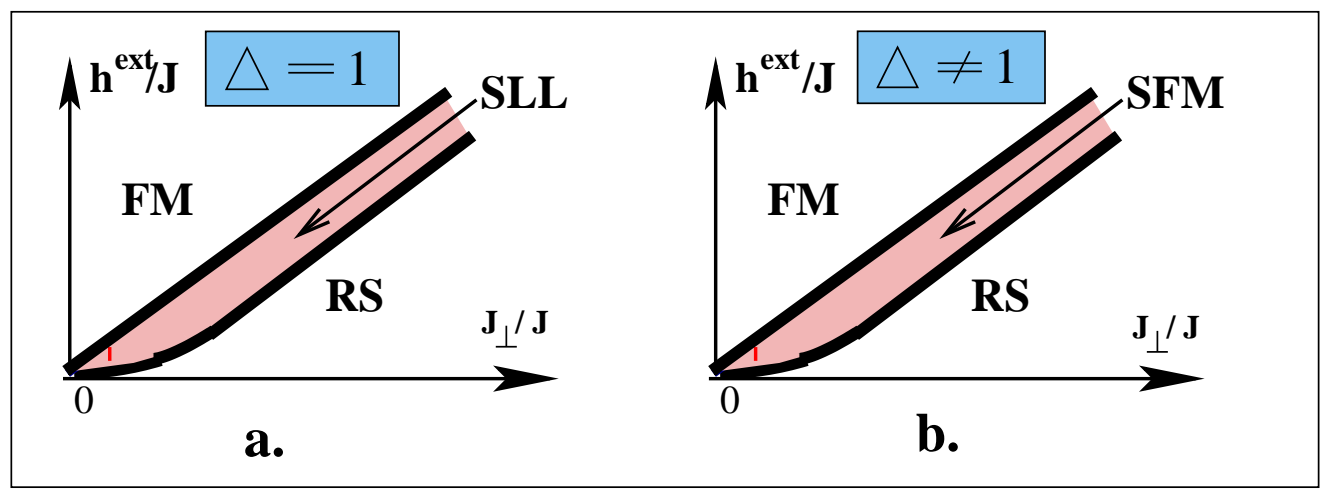

Figure 2. Qualitative sketch of the weak-coupling phase diagram of a two-leg ferromagnetic ladder as a function of the applied magnetic field $\left(\mathrm{h}^{e x t}\right)$ and the isotropic interleg coupling $\left(J_{\perp}\right)$ [29]. In the case of isotropic spin-exchange interaction in legs $(\Delta=1)$ the spin gapped Rung-Singlet (RS) phase is separated from the fully polarized ferromagnetic (FM) phase via the gapless Spin-Luttinger-Liquid (SLL) phase. In the case of finite intraleg exchange anisotropy $(\Delta \neq 1)$ the RS phase is splitted from the FM phase by the gapped Stripe-Ferromagnetic (SFM) phase.

the $S_{z}=-1$ component of triplet create a new effective spin $\tau=1 / 2$ system. One can easily project the original ladder Hamiltonian (11) on the new singlet-triplet subspace

$$
|\Uparrow\rangle \equiv|s\rangle=\frac{1}{\sqrt{2}}[|\uparrow \downarrow\rangle-|\downarrow \uparrow\rangle], \quad|\Downarrow\rangle \equiv\left|t^{-}\right\rangle=|\downarrow \downarrow\rangle .
$$

This leads to the definition of the effective spin $1 / 2$ operators

$$
S_{n, \alpha=1,2}^{+}=(-1)^{n+\alpha} \frac{1}{\sqrt{2}} \tau_{n}^{+}, \quad S_{n, \alpha=1,2}^{z}=\frac{1}{4}\left[I+2 \tau_{n}^{z}\right]
$$

The effective Hamiltonian in terms of the effective spin operators (4) up to the accuracy of an irrelevant constant becomes the Hamiltonian of the spin 1/2 fully anisotropic $X Y Z$ chain in an effective magnetic field

$$
H_{\text {eff }}=-J \sum_{i}\left[\frac{1}{2} \tau_{n}^{z} \tau_{n+1}^{z}+\tau_{n}^{y} \tau_{n+1}^{y}+\Delta \tau_{n}^{x} \tau_{n+1}^{x}\right]+\mathrm{h}^{\text {eff }} \sum_{n} \tau_{n}^{z},
$$

where $h^{\text {eff }}=\mathrm{h}^{\text {ext }}-J_{\perp}+J / 2$. Note that in deriving (5), we have used the rotation in the effective spin space which interchanges the $x$ and $z$ axes.

At $\Delta=1$, the effective problem reduces to the theory of the $X X Z$ chain with a fixed ferromagnetic $X Y$ anisotropy of $1 / 2$ in a magnetic field, which allows for rigorous analysis [29]. The gapped phase at $\mathrm{h}^{\text {eff }}<\mathrm{h}_{c 1}^{\text {eff }}$ for the ladder corresponds to the negatively saturated magnetization phase for the effective spin chain, whereas the massless phase for the ladder corresponds to the finite magnetization phase of the effective spin- $1 / 2$ chain. The critical field $h_{c 2}^{e f f}$ corresponds to the fully magnetized phase of the effective spin chain where the ladder is totally magnetized. From the exact ground state phase diagram of the anisotropic $X X Z$ chain in a magnetic field [43], it is easy to check that $\mathrm{h}_{c 1, c 2}^{e f f}=\mp J / 2$. For the isotropic ferromagnetic ladder in a magnetic field this corresponds to a transitions from rung-dimers to SLL phase at $\mathrm{h}_{c 1}^{e x t}=J_{\perp}-J$ and a 
transition from SLL phase into the fully polarized phase at $\mathrm{h}_{c 2}^{e x t}=J_{\perp}$ (see Fig $2 \mathrm{a}$ ). In this paper we compare this values of critical fields with the numerical results of exact diagonalization method.

Away from the isotropic point $\Delta=1$ the effective Hamiltonian (5) describes the fully anisotropic ferromagnetic $X Y Z$, chain in a magnetic field that is directed perpendicular to the easy axis. For the particular value of magnetic field $\mathrm{h}_{\text {eff }}=0$, the effective $X Y Z$ chain is long range ordered in $Y$-direction [44], accordingly the original ladder system being ordered in the direction perpendicular to the applied magnetic field with opposite magnetization on legs (stripe-ferromagnetic phase). For larger values of the effective field it is clear that this SFM order will be replaced either by the rung singlet phase or the phase with only one order parameter - magnetization along the applied field.

The exact diagonalization study of finite ladder systems (up to length $L=12(\mathrm{~N}=$ 24)) show that the magnetic phase diagram of the ferromagnetic ladder with anisotropic legs in a transverse magnetic field really presents two quantum phase transitions with increasing magnetic field. The first transition is from a gapped RS phase to the gapped SFM phase and the second one from a SFM phase into the fully polarized FM phase (see Fig, $2 \mathrm{~b}$ ).

\section{Exact diagonalization results}

In order to explore the nature of the spectrum and the phase transition, we use the modified Lanczos method [41, 45] to diagonalize numerically finite $(N=12,16,20,24$ sites) ladder systems. The energies of the few lowest eigenstates were obtained for chains with periodic boundary conditions.
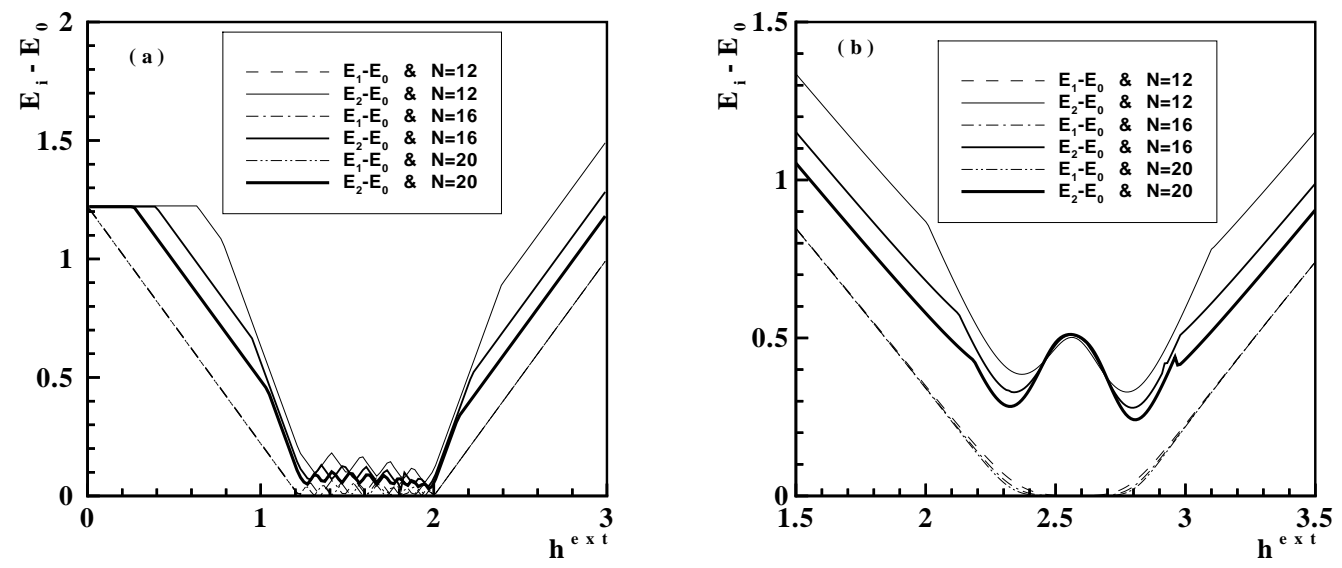

Figure 3. Difference between the energy of the two lowest levels and the ground state energy as a function of the magnetic field, (a) $\mathrm{h}^{e x t}$ for $J_{\perp}=2 J$ and $\Delta=1.0$ and (b) for $J_{\perp}=3 J$ and $\Delta=0.5$ including different ladder lengths $N=12,16,20$.

To show, that the transition lines can be easily observed from the numerical 
calculations of small systems we start our consideration by the case of isotropic chains. In this case, the magnetic field term commutes with the spin-spin exchange terms which makes the total x-component $\left(S_{t o t}^{x}\right)$ of spin a conserved quantity. Thus, the energy levels of ladder are labeled by $S_{t o t}^{x}$. We have computed the vlaue of energy levels in the absence of magnetic field and added the effect of magnetic field by $-J \mathrm{~h}^{\text {ext }} S_{t o t}^{x}$. This makes very high accuracy for the value of energy levels. In Fig 3 a we have plotted the three lowest levels of $N=12,16,20$ ladder with $J_{\perp}=2 J$ as a function of the external field $h^{e x t}$. We determine the excitation gap in the system as the difference between the first excited state and the ground state. As it is clearly seen from this figure in the case of zero magnetic field the spectrum of the model is gapped. For $\mathrm{h}^{\text {ext }} \neq 0$ the gap decreases linearly with $\mathrm{h}^{e x t}$ and vanishes at the critical field, $\mathrm{h}_{c 1}^{e x t}$. This is the first level crossing between the ground state energy and the first excited state one. To get an accurate estimate of $h_{c 1}^{e x t}$ we have obtained the first level crossing for system sizes of $N=12,16,20,24$. The finite size behaviour of this values leads us to $\mathrm{h}_{c 1}^{e x t}=1.2 \pm 0.01$ for $N \rightarrow \infty$. The spectrum remains gapless for $\mathrm{h}_{c 1}^{e x t}<\mathrm{h}^{e x t}<\mathrm{h}_{c 2}^{e x t}$ and becomes once again gapped for $\mathrm{h}^{e x t}>\mathrm{h}_{c 2}^{e x t}=2.0$. We got $\mathrm{h}_{c 2}^{e x t}=2.0$ as an exact value since there was no finite size correction at this value. We should mention that the critical field values obtained in the previous section come from the first order (perturbation) effective Hamiltonian approach and are not exact values. However, the values of the critical fields $\mathrm{h}_{c 1}^{e x t}$ and $\mathrm{h}_{c 2}^{e x t}$ obtained from studies of the finite chains are very close to the values get in the previous section. With increasing field, for $\mathrm{h}^{e x t} \gg J_{\perp}, J$ the gap increases linearly with $\mathrm{h}^{\text {ext }}$.

It can be seen in Fig, 3 a for the gapless sector of the phase diagram at $\mathrm{h}_{c 1}^{e x t}<$ $\mathrm{h}^{e x t}<\mathrm{h}_{c 2}^{e x t}$ the two lowest states cross each other $N / 2$ times, that the first crossing occurs at point $\mathrm{h}_{c 1}^{e x t}$ and the last crossing occurs at the point $\mathrm{h}_{c 2}^{e x t}$. In this region we also observe numerous additional level crossing between the lowest second and third eigenstates. These level crossings lead to incommensurate effects that manifest themselves in the oscillatory behavior of the spin correlation functions. All crossings disappear at $\mathrm{h}^{\text {ext }}>\mathrm{h}_{c 2}^{\text {ext }}$ and the correlation functions do not contain oscillatory terms in this region of the phase diagram.

In marked contrast with the isotropic case, the similar analysis of the few lowest levels for an anisotropic ladder in the presence of transverse magnetic field reveal a principally different behaviour. The energy difference between the two lowest levels and the ground state energy as a function of the magnetic field $\mathrm{h}^{\text {ext }}$ has been computed for $J_{\perp}=3 J$ and $J=1.0$ for different ladder lengths $N=12,16,20$ and different values of the anisotropy parameter $\Delta=0.3,0.5,0.7$. As an example, in Fig $3 \mathrm{~b}$ we have plotted results of this calculations for $\Delta=0.5$. As it is seen from the figure, the excitation spectrum in this case is gapfull except at the two critical points $\mathrm{h}_{c 1}^{e x t}=2.3 \pm 0.1$ and $\mathrm{h}_{c 2}^{e x t}=3.0 \pm 0.1$. In the region of magnetic fields $\mathrm{h}_{c 1}^{e x t}<\mathrm{h}^{e x t}<\mathrm{h}_{c 2}^{e x t}$ the two lowest states form a twofold degenerate ground state in the thermodynamic limit and the spin gap, which appear at $\mathrm{h}^{e x t}>\mathrm{h}_{c 1}^{e x t}$, first increase vs external field and after passing a maximum decreases to vanishe at $\mathrm{h}_{c 2}^{e x t}$. At $\mathrm{h}^{e x t}>\mathrm{h}_{c 2}^{e x t}$ the gap once again opens and, for a 
sufficiently large field becomes proportional to $\mathrm{h}^{e x t}$. These results are in good agreement with the results obtained in the studies of the fully anisotropic antiferromagnetic $X Y Z$ chain in a magnetic field [40].

To determine the properties of this model in different sectors of the phase diagram we have implemented the modified Lanczos algorithm of finite size ladders $(N=$ $12,16,20,24)$ to calculate the magnetizations $M^{x, y, z}$ and spin structure factors $\mathcal{S}^{x x}(q)$, $\mathcal{S}^{y y}(q)$ and $\mathcal{S}^{z z}(q)$.
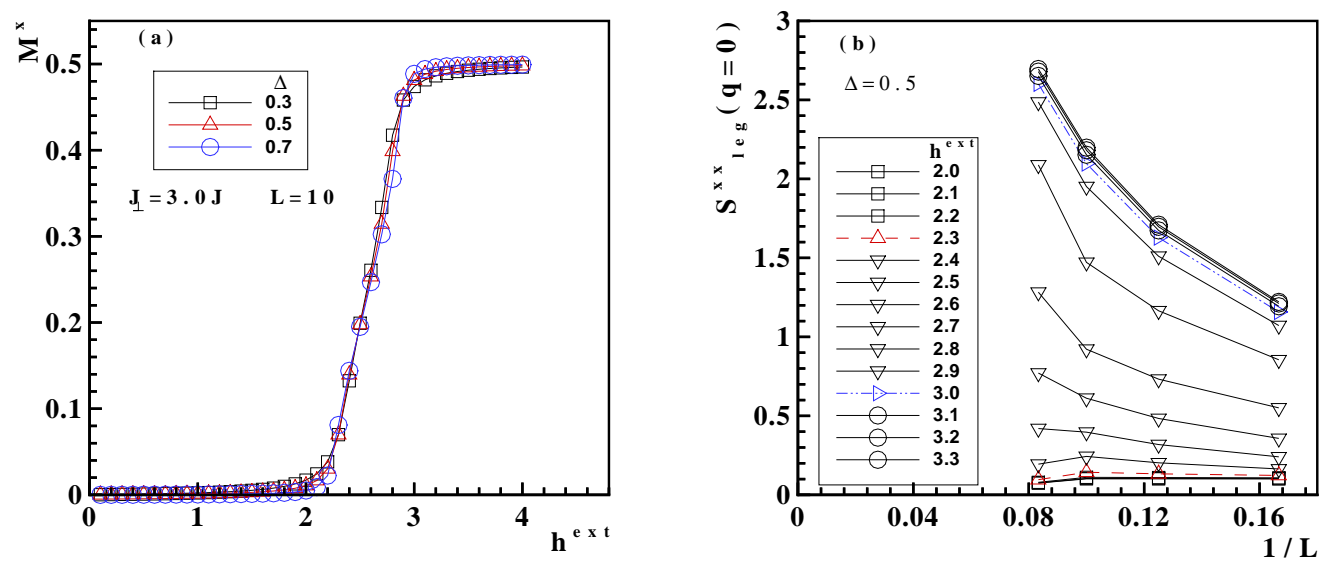

Figure 4. a. The transverse magnetization $M_{x}$ as a function of applied field $\mathrm{h}^{\text {ext }}$ for $L=10$ ladder with $J_{\perp}=3.0 \mathrm{~J}$ and for different values of the anisotropy parameter $\Delta=0.3,0.5,0.7$. b. The intraleg spin structure factor in the $\mathrm{x}$ direction at $q=0$ for $J_{\perp}=3.0 \mathrm{~J}$ and $\Delta=0.5$ plotted as a function of the inverse ladder length $L(L=N / 2$ ) for different strength of the applied field $h^{\text {ext }}$. The dashed line which corresponds to the $h^{e x t}=h_{c 1}^{e x t}=2.3 J$ marks the transition from the RS phase to the intermediate phase with finite magnetization along the field and the dashed-dotted line corresponds to $h^{e x t}=h_{c 2}^{e x t}=3.0 \mathrm{~J}$ and marks the transition into the phase with full magnetization along the field.

In Fig 4 a we have plotted the magnetization along the applied transverse magnetic field, $M^{x}$ vs h ${ }^{e x t}$ for $N=20, J_{\perp}=3 J$ and for different values of the anisotropy parameter $\Delta=0.3,0.5,0.7$. Due to the profound effect of quantum fluctuations the transverse magnetization remains small but finite for $0<\mathrm{h}^{\text {ext }}<\mathrm{h}_{c 1}^{e x t}$ and reaches zero at $\mathrm{h}^{\text {ext }}=0$. This is in agreement with results obtained within the weak-coupling continuum-limit bosonization treatment [29] and with the results for the magnetization obtained in the case of fully anisotropic $X Y Z$ chain [40]. For $\mathrm{h}^{\text {ext }}>\mathrm{h}_{c 1}^{\text {ext }}$ the magnetization increases linearly with increasing field once again in complete agreement with the predictions of bosonization analysis [29]. This behavior is in agreement with expectations, based on the general statement that in the gapped rung-singlet phase, magnetization along the applied field appears only at a finite critical value of the magnetic field equal to the spin gap [13-16]. However, in finite systems we do not observe a sharp transition at this point $\left(\mathrm{h}_{c 1}^{e x t}\right)$ or close to the saturation value which happens at $\mathrm{h}^{e x t}>\mathrm{h}_{c 2}^{e x t}$. Magnetization along the directions perpendicular to the applied field remains small, but finite. However, as 


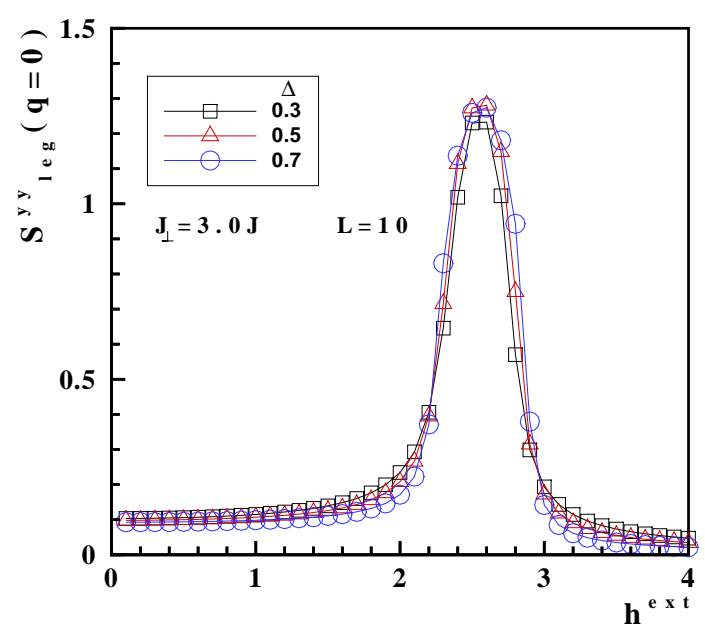

Figure 5. The intraleg spin structure factor $\mathcal{S}_{\text {leg }}^{y y}(q=0)$ plotted as a function of the applied field $\mathrm{h}^{\text {ext }}$ for $L=10$ ladder with $J_{\perp}=3.0 \mathrm{~J}$ and for different values of the anisotropy parametere $\Delta=0.3,0.5,0.7$.

we will show below, for $\mathrm{h}_{c 1}^{\text {ext }}<\mathrm{h}^{\text {ext }}<\mathrm{h}_{c 2}^{\text {ext }}$ the intraleg magnetization along the "y" direction is finite and shows the long-range ferromagnetic order.

An additional insight into the nature of different phases can be obtained by studying the spin-spin correlation functions. In particular we study the magnetic field dependence of the different spin structure factors. We have calculated the intraleg spin structure factors defined by

$$
\mathcal{S}_{l e g}^{\alpha \beta}(q)=\frac{1}{N} \sum_{n, r}\left\langle 0\left|s_{j, n}^{\alpha} s_{j, n+r}^{\beta}\right| 0\right\rangle e^{i q r} .
$$

The field dependence of the intraleg spin structure factor $\mathcal{S}_{\text {leg }}^{x x}(q=0)$ is qualitatively the same as of the transverse magnetization $M_{x}$. In Fig $4 \mathrm{~b}$ we have plotted the intraleg spin structure factor $\mathcal{S}_{\text {leg }}^{x x}(q=0)$ for different strength of the applied magnetic field chosen in the vicinity of $h_{c 1, c 2}^{e x t}$ as a function of the ladder length $L$. As it is seen from this figure the dashed line which corresponds to the $h^{e x t}=h_{c 1}^{e x t}=2.3 \pm 0.1$ marks the transition from the RS phase to the intermediate phase with finite magnetization along the field and the dash-dotted line which corresponds to $h^{e x t}=h_{c 2}^{e x t}=3.0 \pm 0.1$ marks the transition into the phase with saturate magnetization along the field.

In Fig 5 we have plotted $\mathcal{S}_{\text {leg }}^{y y}(q=0)$ as a function of the applied field $\mathrm{h}^{\text {ext }}$ for $L=10, J_{\perp}=3 J$ and different values of the anisotropy parameter $\Delta=0.3,0.5,0.7$. As it is clearly seen from this figure, in complete agreement with the bosonization results [29], there is not any long range ferromagnetic order along the " $y$ " direction at $\mathrm{h}^{\text {ext }}<\mathrm{h}_{c 1}^{\text {ext }}=2.3 \pm 0.1$ and $\mathrm{h}^{\text {ext }}>\mathrm{h}_{c 2}^{\text {ext }}=3.0 \pm 0.1$. However, in the intermediate region $\mathrm{h}_{c 1}^{e x t}<\mathrm{h}^{e x t}<\mathrm{h}_{c 2}^{e x t}$, the spins of each leg show a profound ferromagnetic order in the " $y$ " direction.

To display the magnetic phase diagram of the anisotropic ferromagnetic ladder 
in a transverse magnetic field we have also calculated numerically the intrarung spin correlation functions. We have computed the on-rung dimerization order parameter given by

$$
d_{r}=\frac{1}{N} \sum_{n}\left\langle 0\left|\vec{s}_{1, n} \cdot \vec{s}_{2, n}\right| 0\right\rangle .
$$

In Fig. 6a we have plotted $d_{r}$ as a function of $\mathrm{h}^{\text {ext }}$ for the ladder with $J_{\perp}=3 J, \Delta=0.5$ and for different values of the ladder lengths $N=12,16,20,24$. As it is clearly seen from this figure for $h^{e x t}<h_{c 1}^{e x t}, d_{r}$ is close to -0.75) and the ladder is in the rung singlet phase. For $h^{e x t}>h_{c 2}^{e x t}, d_{r}$ is slightly less then the saturation value $d_{r} \sim 0.25$ and the ferromagnetic long range order along the " $x$ " axis is present. Deviation from the saturation values -0.75 and 0.25 is the result of quantum fluctuations. In this case of finite systems and with chosen values of the rung exchange, the critical magnetic fields are high which strongly suppresses the quantum fluctuations. As a result the obtained averages of spin correlation functions are very close to their classical saturation values. In the intermediate range of field the system smoothly evaluates from a RS phase into the FM phase via formation of the stripe-ferromagnetic order.
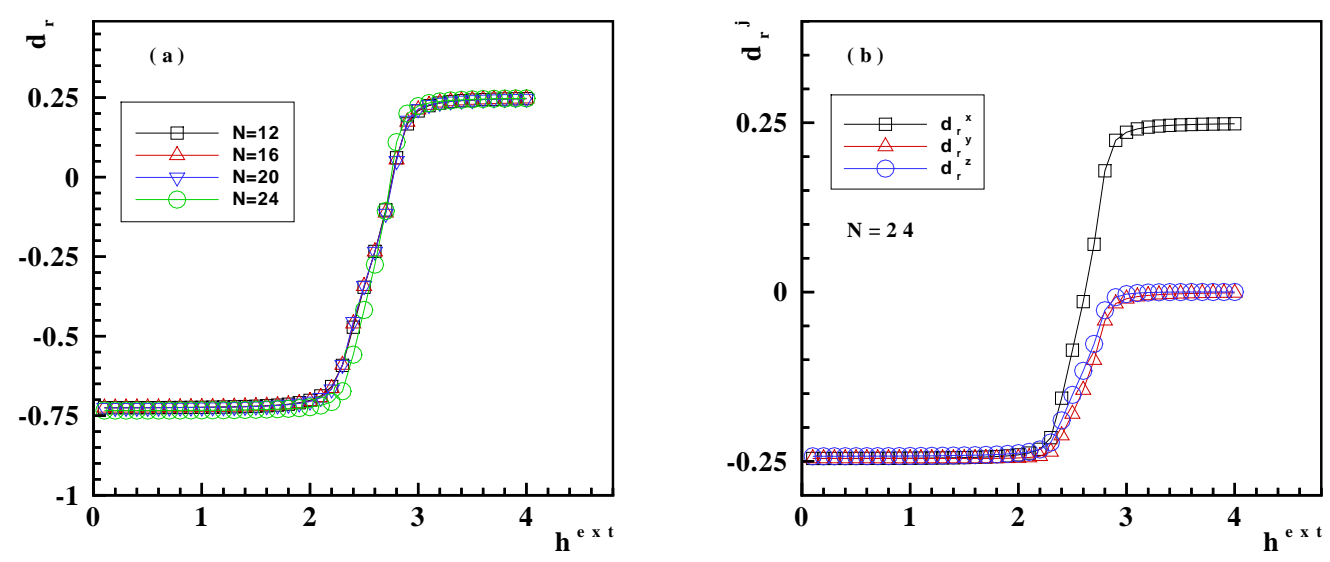

Figure 6. a. The rung-singlet order parameter $d_{r}$ as a function of the applied magnetic field $h^{e x t}$ for the ladder with $J_{\perp}=3.0 J, \Delta=0.5$ and for different values of the ladder length $N=12,16,20,24$. b. The rung-singlet order parameter $d_{r}^{x}, d_{r}^{y}$ and $d_{r}^{z}$ as a function of the applied magnetic field $h^{e x t}$ for the ladder with $J_{\perp}=3.0 \mathrm{~J}, \Delta=0.5$ and $N=24$.

To get additional data about the character of the spin ordering in the intermediate SFM phase we have calculated the " $x ", " y$ " and " $z$ " components of the rung-singlet order parameter given by

$$
d_{r}^{j}=\frac{1}{N} \sum_{n}\left\langle 0\left|s_{1, n}^{j} s_{2, n}^{j}\right| 0\right\rangle, \quad j=x, y, z .
$$

In Fig 6b we have plotted $d^{x}, d^{y}$ and $d^{z}$ as a function of the applied field $\mathrm{h}^{\text {ext }}$ for $N=24$ ladder with $J_{\perp}=3 J$ and for $\Delta=0.5$. As it is seen from this figure in 
the RS, at $\mathrm{h}^{e x t}<\mathrm{h}_{c 1}^{e x t}$ the system clearly shows the $S U(2)$ invariant order within the rung $d^{x}=d^{y}=d^{z} \sim-0.25$. For $\mathrm{h}^{e x t}>\mathrm{h}_{c 1}^{e x t}$ the $S U(2)$ invariance is broken, $d^{x}$ increases with increasing field faster then the other two components and soon approaches the positive values corresponding to the ferromagnetic order in the " $x$ " direction, while $d^{y}=d^{z}$ remain negative indicating the antiferromagnetic correlations in these components. This shows the suppressed ferromagnetic order in the " $z$ " direction and profound ferromagnetic response of the " $y$ " direction presents completely convincing arguments in support of statement, that for intermediate values of applied field, at $\mathrm{h}_{c 1}^{e x t}<\mathrm{h}^{e x t}<\mathrm{h}_{c 2}^{e x t}$ ferromagnetic ordering in the " $x$ " direction of spin from both legs is accompanied by antiferromagnetic order in the " $y$ " direction of spins located on different legs and completely suppressed correlations in the " $z$ " direction. It corresponds to the stripe ferromagnetic phase. Finally for $\mathrm{h}^{\text {ext }}>\mathrm{h}_{c 2}^{e x t}$ the ordering in " $y$ " and " $z$ " directions are completely suppressed and the system shows ferromagnetic order with nominal magnetization per spin along the applied field.

\section{Conclusions}

We have studied the ground state phase diagram of the two-leg spin ladders with ferromagnetic anisotropic legs in a transverse magnetic field. We have implemented the modified Lanczos method to get the excited state energies as the same accuracy of the ground state one. Two quantum phase transitions in the ground state of the system with increasing magnetic field have been identified. The first transition is the gapped rung-singlet to the gapped stripe-ferromagnetic phase. The second - to the transition from the gapped stripe-ferromagnetic phase into the fully polarized ferromagnetic phase. This results are in complete agreement with the results obtained from the bosonization treatment 29].

\section{Acknowledgments}

It is our pleasure to thank D. Cabra, H.-J. Mikeska, E. Pogosyan and T. Vekua for fruitful discussions. GIJ would like to thank A. Ferraz for interesting discussions and kind hospitality during his stay in ICCMP-UnB. He also acknowledges support from the CNPq and the Ministry of Science and Technology of Brazil and support of the Georgian National Science Foundation (Grant GNSF/ST06/4-018).

\section{References}

[1] E. Dagotto 1999 Rep. Prog. Phys. 62 1525; E. Dagotto and T.M. Rice 1996 Science 271, 618 (1996).

[2] Schmidt K P and Uhrig G S, 2005 Mod. Phys. Lett. b 191179

[3] Chaboussant G, Crowell P A, Lèvy L P, Piovesana O, Madouri A and Mailly D 1997 Phys. Rev. $B \mathbf{5 5}, 3046$ 
[4] Chaboussant G, Julien M-H, Fagot-Revurat Y, Hanson M, Lèvy L P, Berthier C, Horvatic M and Piovesana O 1998 Europ. Phys. J. B 6167

[5] Chaboussant G, Fagot-Revurat Y, Julien M-H, Hanson M E, Berthier C, Horvatic M, Lèvy L P, and Piovesana O 1998 Phys. Rev. Lett. 802713

[6] Arcon D, Lappas A, Margadonna S, Prassides K, Ribera E, Veciana J, Rovira C, Henriques R T and M. Almeida M, 1999 Phys. Rev. B 604191

[7] Mayaffre H, Horvatic H, Berthier C, Julien M-H, Söransan P, Lèvy L P, and Piovesana O 2000 Phys. Rev. Lett. 854795

[8] Watson B C, Kotov V N, Meisel N W, Hall D W, Granroth G E, Montfrooij W T, Nagler S E, Jensen D A, Backov R, Petruska M A, Fanucci G E and Talham D R, 2001 Phys. Rev. Lett. 86, 5168

[9] Schulz H J, 1986 Phys. Rev. B 346372

[10] Shelton D G, Nersesyan A A and Tsvelik A M, 1996 Phys. Rev. B 53, 8521

[11] Hawyard C, Poilblanc D, and Lèvy L P, 1996 Phys. Rev. B 5412649

[12] Chitra R and Giamarchi T, 1997 Phys. Rev. B 55, 5816

[13] Cabra D C, A. Honecker A, and Pujol P, 1997 Phys. Rev. Lett. 79, 5126; -1998 Phys. Rev. B 58, 6241

[14] Totsuka K, 1998 Phys. Rev. B 57345

[15] Usami M and Suga S-i, 1998 Phys. Rev. B 5814401

[16] Mila F, 1998 Eur. Phys. J. B 6201

[17] Giamarchi T and Tsvelik A, 1999 Phys. Rev. B 59, 11398

[18] Furusaki A and Zhang S C, 1999 Phys. Rev. B 601175

[19] Normand B, Kyriakidis and Loss D, 2000 Ann. Phys. (Leipzig) 9133

[20] Wang X and Yu L 2000 Phys. Rev. Lett. 845399

[21] Hagiwara H, Katori H A, Schollwök U and Mikeska H-J, 2000 Phys. Rev. B 621051

[22] Wessel S, Olshanii M and Haas S, 2001 Phys. Rev. Lett. 87206407

[23] Chitra R and Citro R 2001 Phys. Rev. B 63054441

[24] Wiessner R M, Fledderjohann A, Mutter K -H and Karbach M, 1999 Phys. Rev. B 606545

[25] Hikihara T and Furusaki A 2001 Phys. Rev. B 63134438

[26] Kolezhuk A K and Mikeska H-J, 1998 Int. J. Mod. Phys. 12, 2325

[27] Sakai T and Takahashi M, 1998 Phys. Rev. B 57, 8091

[28] Vekua T, Japaridze G I, and H.-J. Mikeska H-J, 2003 Phys. Rev. B 67064419

[29] Vekua T, Japaridze G I, and H.-J. Mikeska H-J, 2004 Phys. Rev. B 70014425

[30] Japaridze G I, Nersesyan A A and Wiegmann P B, 1984 Nucl. Phys.B 230 (FS 4), 511

[31] Japaridze G I and Nersesyan A A, 1978 Sov. Phys. JETP Lett. 27 334; Pokrovsky V L and Talapov A L, 1979 Phys. Rev. Lett. 4265

[32] Mikeska H-J, 1978 J. Phys. C: Solid St. Phys. 11, L29.

[33] Hieida H, Okunishi K and Akutsu Y, 2001 Phys. Rev. B 64224422

[34] Dmitriev D V, Krivnov V Y and Ovchinnikov A A, 2002 Phys. Rev.B 65, 172409 (2002);

[35] Dmitriev D V, Krivnov V Y, Ovchinnikov A A and Langari A, 2002 JETP 95538

[36] Dutta A and Sen D, 2002 Phys. Rev. B 67094435

[37] Caux J-S, Essler F H L and Löw U, 2003 Phys. Rev. B 68134431

[38] Langari A, 2004 Phys. Rev. B 69, 100402(R)

[39] Dmitriev D V and Krivnov V Y, 2004 Phys. Rev. B 70144414

[40] Hogemans R, Caux J-S and Löw U, 2005 Phys. Rev. B 71014437

[41] Langari A and Mahdavifar S, 2006 Phys. Rev. B 73, 54410

[42] Sachdev S and Bhatt R N, 1990 Phys. Rev. B 419323

[43] Takahashi M, 1999 Thermodynamics of one-dimensional solvable models, (Cambridge: Cambridge University Press) Chapter IV.

[44] Johnson J D, Krinski S and McCoy B 1073 Phys. Rev. A 82526

[45] Grosso G and Martinelli L, 1995 Phys. Rev. B 5113033. 